\title{
La sociofenomenología de A. Schütz: entre el constructivismo y el realismo
}

\author{
M. Carmen López Sáenz \\ Profesora de Filosofia. Universidad de la Rioja. Edificio "Luis Vives" \\ c. Luis de Ulloa. 26004 Logrońo (La Rioja). Spain
}

\section{Resumen}

En este artículo valoramos la relación del pensamiento de A. Schütz con la fenomenología de Husserl y Merleau-Ponty y afirmamos que, a pesar de su problematicidad, Schütz es el padre de la sociofenomenologia, ya que fue capaz de aplicar la filosofia de Husserl al análisis social.

Schürz parrió de los estudios hussertianos sobre la Lebenswelt, de su psicología eidética y aplicó el método de la fenomenologfa transcendensal a sus trabajos sobre el mundo de la vida cotidiana. Esta vinculación con la fenomenologia puede ejemplificarse en el tratamiento schütziano de la intersubjetividad y en su filosofia de las ciencias humanas y sociales.

Después de realizar este examen, evaluamos críticamente la tendencia contemporánea a clasificar la fenomenologia entre los idealismos y el pensamiento de Schürz entre las teorias constructivistas de la realidad social y llegamos a la conclusión de que ambas afirmaciones son excesivamente simplistas e inadecuadas.

Palabras clave: sociofenomenología, intersubjetividad, constructivismo, realismo, idealismo.

Abstract. A. Schiitz sociophenomenology: between constructivism and realism

This article examines the relationship berween A. Schütz's thought and Phenomenology. Despite its controversy, Schürz is the creator of the Sociophenomenology, as he was able to apply Husserl's Philosophy to the social analysis.

Schüz Husserl' investigations about Lebenswelt, and its eidetics $P_{\text {sychology. He applied }}$ the method of the transcendental Phenomenology in his worked on the wotld of the daily life. This linking with the Phenomenology can be illustrated in Schütz's treatment of Intersubjectivity and his philosophy of the human and social sciences.

Once this examined, we can assert a contemporary tendency to critically classify Phenomenology as a type of idealism and Schürz's thought amongst constructivist theories of social reality. We conclude thar both assertions are too over-simple and inappropriate.

Key words: Sociophenomenology, intersubjectivity, constructivism, realism, idealism. 


\begin{aligned} & \multicolumn{2}{c}{ Sumario } \\ & La fundamentación filosofica Constructivismo, realismo \\ & de la sociología comprensiva o idealismo fenomenologico \\ & Intersubjetividad como fundamento Conclusion \\ & de la sociabilidad \end{aligned}

\section{La fundamentación filosófica de la sociología comprensiva}

Alfred Schutz (1899-1959) es, sin duda, el máximo representante de la sociofenomenología. A pesar de que su rica producción tiende más a dilucidar los conceptos de otros pensadores como Husserl, Weber, etc. que a producir análisis independientes, su mérito principal es el descubrimiento de la importancia de la fenomenología en los estudios sociológicos y la creación de una sociología que investiga la estructura y la distribución social del conocimiento de sentido común. La sociofenomenología de Schütz pretende ser más una descripción de la experiencia de la actitud natural que un análisis de ella desde una perspectiva fenomenológico-transcendental. Para ello, Schütz completa la fenomenología husserliana con la sociología de la acción y la comprensión de Max Weber e intenta hacer compatibles la neutralidad valorativa weberiana y la aprehensión del sentido de los comportamientos proclamada por la fenomenología.

Siguiendo a Weber, Schütz piensa que el tema principal de las ciencias sociales son los actores sociales y que su conducta no puede explicarse mediante esquemas causales, sino que tiene que ser comprendida. De ahi su adhesión a la sociología comprensiva. Sin embargo, Schütz critica a Weber por no analizar la formación intersubjeriva del sentido que los actores dan al mundo social. Piensa que el concepto weberiano de acción significativa tiene ciertas inadecuaciones, ya que no presupone un análisis detallado del significado y de la acción significativa ordinaria. En definitiva, Schütz quiso corregir la falta de fundamentación filosófica de los conceptos weberianos y consideró que la comprensión subjetiva exigía conocimiento de las funciones conscientes del hombre. Weber se había limitado a desarrollar los instrumentos necesarios para sus estudios empíricos, pero no había profundizado en la comprensión del significado subjetivo de la acción social, en la intersubjetividad o en las estructuras de la conciencia a las que se vinculan los significados. Con la pretensión de analizar estos principios y fundamentar filosóficamente las ciencias sociales, Schütz se dirigió a Husserl en los años veinte y, entre junio de 1932 y diciembre de 1937, se entrevistó con él. Husser le enseñó que la vuelta a las cosas mismas requería un análisis de la subjetividad que constituye el mundo objetivo y es, a su vez, constituida por él. Sin embargo, Schütz se interesó más por la fenomenología de la actitud natural que por la fenomenología transcendental. Concebía la sociología como una ciencia que produce construcciones de segundo grado derivadas de las que tienen lugar en la vida cotidiana y estaba convencido de que la fenomenología, en tanto método que se enfrenta a la realidad vivenciada, podía enriquecer a la sociología comprensiva. 
Si el sujeto de la ciencia, en opinión de Husserl, era la intersubjetividad transcendental, el universo de mónadas que precedia y producía toda la objetividad mundana, el sujeto de la ciencia de Schütz es el científico individual y su comunidad. No obstante, está de acuerdo con Husserl en que el mundo de la teoría científica es tan sólo una provincia finita de significado en medio de otros mundos actuales y posibles; además, la finalidad de la ciencia no es alcanzar un mundo de significados objetivos y neutros, porque éste es, para Schütz, una abstracción: el significado siempre es subjetivo, es decir, siempre hace referencia a un sujeto.

Siguiendo a Weber y a Husserl, considera que las ciencias sociales han de alcanzar cientificidad no por imitación de la exactitud del método de las ciencias naturales, sino por la aplicación rigurosa de un método científico más apropiado al objeto de estudio de aquéllas: el método comprensivo, que pretende conocer el significado subjetivo de las acciones humanas. La hermenéutica fenomenológica nos ha enseñado, además, que la prioridad del método amenaza con perder de vista el objeto y la verdad; por tanto, éste no ha de ser ajeno a la cosa investigada. A pesar de que el método fenomenológico haya sido tan fructífero, Husserl no lo desvinculó del objeto fundamental de la filosofía, no lo absolutizó. Por su parte, Schütz adopta la fenomenología como un pensamiento adecuado para fundamentar la teoría weberiana, porque trabaja con la realidad intencional: la realidad queda sometida a la epojé, de manera que nos quedamos con una realidad vivida que actúa como materia prima para el científico social. Pero la epojé es sólo una desconexión, una puesta entre paréntesis que no suprime la verdadera realidad, sino que exige una reducción, entendida como re(con)ducción al yo donador de sentido.

Schütz no cree que las cuestiones fenomenológicas estén enfrentadas con los procedimientos empíricos y opina que el método fenomenológico puede ser de gran utilidad si se aplica a las ciencias sociales. La fenomenología problematiza conceptos como la existencia del hombre social, el significado de los otros, las características comunes de los distintos mundos socioculturales en los que se despliega la realidad histórica. Sin embargo, Schütz selecciona únicamente aquellos aspectos de la fenomenología que le resultan útiles para realizar sus objetivos: los análisis husserlianos de la experiencia pre-predicativa y de la naturaleza de los tipos ${ }^{i}$ la reducción fenomenológica, la reducción eidética, el modelo de la apercepción y la apresentación, la retención y la protención, la nóesis y el noema, la concepción de las cosas como intersubjetivamente idénticas, relativas a una infinidad de sujetos que se hallan en una relación de compren-

1. Aunque los procesos de tipificación actúan como verdaderos a priori, no tienen como objetivo el descubrimiento del eidos. Si para Husserl la esencia es la estructura significativa de la conciencia intencional, para Schütz lo importante no es la búsqueda de la esencia del estado, de la sociedad, erc., sino el examen de los rasgos generales de los fenómenos con objeto de manifestar su múltiple estructura y su génesis formal. La esencia no es, pues, para Schïtz, un concepto metafísico ni un recurso metodológico con determinaciones empíricas. Conviene además tener en cuenta que Husserl no aplicó esos tipos al análisis social. 
sión mutua ${ }^{2}$; acepta además la idea husserliana de la empatía como explicación de la constitución de la objetividad intersubjetiva de las cosas y de los seres humanos como seres psicofísicos unitarios ${ }^{3}$; sin embargo, todos estos conceptos sólo tienen, para Schütz, un valor propedéutico. Al igual que Husserl, se plantea preguntas acerca de la objetividad del mundo; acepta el método eidético para intuir las esencias (lo cual no equivale a crearlas, sino a aprehender activamente lo que esta preconstituido de manera pasiva por el tipo) ${ }^{4}$; además, la teoría de la tipificación de Schütz desarrolla la doctrina husserliana de la intencionalidad en la estructura de la experiencia pre-predicativa y en el proceso de ideación y abstracción que tiene lugar en la actitud natural. De acuerdo con Husserl, piensa que la experiencia es siempre experiencia tipificada y que tal tipificación tiene lugar en el nivel pre-predicativo (nivel de pasividad). Ambos autores comprenden la importancia de la típificación en la experiencia del mundo de la vida; saben que los tipos sirven como punto de partida del método eidético, que lo genérico está ya presente en el nivel de la pasividad. Sin embargo, Schütz aprueba sólo parcialmente la afirmación husserliana de los múltiples niveles de pasividad y considera que las síntesis pasivas, especialmente las de asociación, son formas generales inmanentes a la conciencia; cree que la aserción husserliana de que la identidad del noema se realiza en la sintesis pasiva no es correcta, ya que no podemos asumir un cumplimiento de conciencia de modo pasivo. Además Schüzz abandonó algunos conceptos clave de la fenomenología por considerarlos excesivamente idealistas y acus 6 a Husserl de no prestar atención a los problemas concretos de las ciencias sociales.

En definitiva, Schütz concibe la fenomenología fundamentalmente como tematización de la actitud natural, de la Lebenswelt. Por eso, se centra en el análisis de ésta e intenta aplicarle la fenomenología transcendental; de ahí su interés por la psicología fenomenológica o fenomenología de la actitud natural. Es decir, Schütz sigute al Husserl que establece una psicología eidética capaz de fundamentar la psicología empirica ${ }^{5}$, de este modo, se queda en la esfera mundana y abandona la transcendental, aquélla que se ocupa de todos los problemas constitutivos. Como dice Natanson, Schuitz sólo pretendía hacer una psicología de la actitud natural ${ }^{6}$; estaba convencido de que ésta había sido un área descuidada por los fenomenólogos y de que el origen de lo social estaba en lo natural y no en lo transcendental. Sin embargo, valoraba la metodología de la fenomenología transcendental como vía para dotar de racionalidad y cientificidad a la esfera no reducida de la psicología fenomenológica.

Schütz opina que la aportación más importante de Husserl es su análisis de la Lebenswelt, que orienta el desarrollo de una antropología filosófica, y su

2. Cfr. A. Schürz. Collected Papers III. La Haya: M. Nijhoff, 1966, p. 17.

3. Ibid. p. 26.

4. Cfr. A. Schütz. "Type and Eidos...". Philosophy and Phenomenological Research XX. (1959). p. $147-165$, p. 164 .

5. Cfr. A. Schütz, Collected Papers $1 \mathrm{H}$, p. 4.

6. Cfr. M. Natanson. Anonymyte. A Stzudy in the Philosophy of A. Schütz. Bloomingron: Indiana Univ. Press, 1986, p. 123. 
método aplicable al mundo de la actitud natural ${ }^{7}$. Sin embargo, el empleo que hace del concepto de mundo de la vida está delimitado al mundo de la vida cotidiana, mientras que Husserl distinguía éste de lo que en Experiencia y juicio llamaba experiencia antepredicativa. La Lebenswelt de Husserl era fruto del análisis regresivo que partía de la ciencia y hallaba los fundamentos de ésta mediante la reducción. En cambio, para Schuitz, la vida cotidiana es el punto de partida de todo conocimiento actual o posible. Por tanto, abandona el a priori husserliano del mundo de la vida y se refiere al mundo de la actitud natural en tanto mundo vivenciado; de ahí la existencia de varios mundos o realidades múltiples, en función de las diferentes tensiones de la conciencia; confunde, entonces, el mundo original de la pura experiencia con el mundo de la vida cotidiana y con el mundo de las interpretaciones de sentido común. Considera que el mundo de la vida no es sólo físico, sino principalmente social y que, en consecuencia, no es concebible ninguna ontología de él que no comience con la descripción del yo en interrelación con el otro ${ }^{8}$.

Aunque para Schittz el mundo de la vida cotidiana es sólo un ámbito de significado finito del mundo de la vida, se trata de la realidad suprema, justamente aquélla que Husserl pone entre paréntesis para tematizar sus estructuras y su fundamentación, para reencontrarla de manera autoevidente. Como afirma Martín Algarra, en Schütz, el concepto de mundo de la vida no puede, por tanto, ser entendido en términos ontologicos, sino más bien como una noción psicológica y vitalista?.

El estilo cognoscitivo de este mundo de la vida schütziano se denomina actitud natural y está regido por motivos pragmáticos, es decir, orientados al dominio del mundo más que a su conocimiento. En cambio, Husserl se dirigía a él con una orientación prioritariamente gnoseológica cuyo interés no era en absoluto tecnológico o estratégico, sino, en todo caso, emancipativo.

Aunque Schütz caracteriza a este mundo como mundo del ejecutar (working World) cree, como Husserl, que no es autosuficiente, que la reflexión filosófica y social es imprescindible para interpretarlo, porque éste no es, como puede parecer a primera vista, homogéneo, sino incoherente, sólo parcialmente claro y, en ocasiones, contradictorio.

Husserl y Schütz hablan del carácter derivado de la Lebenswelt de la ciencia, pero no establecen una separación radical entre ambos, ya que no se trata de dos regiones ontológicas diferentes, sino de ámbitos de significado interrelacionados, niveles epistemológicos no excluyentes, ya que incluso el científico social se mueve dentro de varias regiones de significado.

En cuanto al concepto de acción, a pesar de que Schütz lo hereda de Weber, profundiza en él situándolo en el mundo de la vida cotidiana y ligándolo

7. Cfr. A. Schüzz. Collected Papers 1. La Haya: M. Nijhoff, 1962, p. 149.

8. Cfr. A. Schütz, "El problema de la intersubjetividad transcendental en Husserl». AA.VV. Huserl. Tercer coloquio filosbfico de Royaumont. Buenos Aires: Paidos, 1968, p. 310.

9. M. Martín Algarra. La comunicación en la vida cotidinna. La fenomenologia de A. Schütz. Pamplona: EUNSA, 1993, p. 253. 
-como haría la fenomenología - a la percepción y a la relevancia de las realidades experimentadas por el sujeto. Esta está estrechamente vinculada al acervo de conocimiento de cada uno, constituido por la tipificación y la sedimentación de experiencias no familiares; la interpretación de las vivencias no es más que el proceso de ordenarlas por medio de su inclusión en ese acervo de conocimiento. Husserl, en cambio, va más allá de ese conjunto de sedimentaciones adquirido y se remonta al origen de esas pautas automáticas de conocimiento, al yo apodíctico que constituye todo significado actual o posible. Nosotros creemos que no es posible prescindir de esta fundamentación transcendental, entre otras razones porque sólo desde ella podremos establecer principios normativos que guíen las acciones y sirvan para contrastar los prejuicios y precomprensiones del sentido común y para distinguir las interpretaciones verdaderas de las falsas.

En ocasiones, da la impresión de que Schütz confunda el papel de los procesos activos y pasivos. Esta dificultad va acompañada de una indistinción entre los aspectos noéticos y noemáticos de los procesos intencionales y, por consiguiente, de la intencionalidad misma. Comenzaremos apuntando la confusión entre las actividades pasivas y las activas. Schütz afrrma que nuestro esquema interpretativo se constituye pasivamente y que no es resultado del pensamiento propio. No nos explica, sin embargo, en qué consiste concretamente esta constitución pasiva, ni cómo son extraídos de la experiencia los esquemas de interpretación. ¿Cómo puede una relevancia impuesta transformarse en relevancia intrínseca? No basta decir que las interpretaciones acrivas se fundan en síntesis pasivas. Las relevancias son formas de tipificación. Sin embargo, la teoria de la tipificación de Schütz no es completa. ¿Las tipificaciones se producen únicamente en el nivel pre-predicativo?, es decir, ¿se constituyen sólo pasivamente? En opinión de Schütz, hay interrelación entre el interés y la relevancia motivacional: aquello que dirige nuestra atención, aquello que nos motiva es aquello que es relevante para nosotros. Mientras que el problema del interés es indisociable de los procesos mentales activos, la teoria de la relevancia de Schütz no establece claramente la distinción entre procesos activos y pasivos, ni clarifica su papel en el fenómeno de la televancia.

Schütz interpreta la acción social como comportamiento humano y la hace consistir en intervención positiva en determinada situación o en conocimiento pasivo de la misma; es decir, acción no es, para él, sinónimo de práctica, sino que también la teoría puede entenderse como tal. Esto es así porque Schütz define la acción como la conducta humana proyectada por el actor de manera autoconsciente. El término acto designa la acción ya realizada. La acción puede ser manifiesta o latente. Toda acción manifiesta es fruto de un proyecto y responde a un propósito. Tomando como punto de partida el postulado de Max Weber referido a la interpretación subjetiva, Schütz se centra en la comprensión de la acción social como sentido que el actor asigna a sus acciones. La interpretación subjetiva de este sentido es, para Schütz, una tipificación del mundo de sentido común, la manera concreta en que los hombres en su vida diaria interpretan su conducta y la de los demás. 
Digamos, para finalizar, que Schütz fue frel a la fenomenología en tanto que se interesó por las estructuras significativas; sin embargo, al final de su vida constató que, aunque la fenomenología clarifica las estructuras de significado para fundamentarlas en la estructura del ser, pretende fundamentar el mundo social sin establecer una ontología de él ${ }^{10}$.

\section{Intersubjetividad como fundamento de la sociabilidad}

Schütz estudió los fundamentos psicológicos de la acción para comprender el significado de la acción social y, para ello, conjugó el concepto de acción social de Weber con el concepto de intersubjetividad de Husserl. Como aquél, pensaba que el mundo de la vida cotidiana era el ámbito común de la intersubjetividad y de la comunicación, pero Schürz acudió a la filosofía para profundizar en el problema de la intersubjetividad.

Si la acción social se define como aquélla que está dirigida hacia otras personas (seres conscientes) por la intención del actor, será necesario primero describir cómo accedemos a dichos seres. Schütz estaba convencido de que el problema básico de la fenomenología eta el de la intersubjetividad y sugería que debería abordarse como una categoría ontológica fundamental de la existencia humana, como una precondición de cualquier experiencia inmediata en el mundo de la vida, como una cuestión filosófica y un problema sistemático para las ciencias sociales.

En la actitud natural, la intersubjetividad no se presenta como problema, sino como hecho evidente ${ }^{11}$. Esta aproblematicidad se debe, en nuestra opinión, a que nos limitamos a aprehender al otro como objeto peculiar, como presentación; en cambio, Husserl estudió profundamente la intersubjetividad porque consideraba que lo que hace sujeto al otro es justamente lo apresentado en él. El pensamiento de sentido común no es completamente adecuado para afrontar el problema de la intersubjetividad, porque en él sólo tenemos la posibilidad de comprender la acción del otro de manera suficiente para nuestros propósitos a mano. Con el fin de aumentar esa comprensión de la alteridad, debemos analizar el sentido que tiene la acción para el actor. Esta interpretación subjetiva del sentido sólo es factible si se revelan los motivos que determinan el curso de su acción. Schütz no aboga por un simple conocimiento de sentido común, sino que distingute la realidad de la tipicidad ideal. Aunque ésta se halla enraizada en aquélla, la fuente de la tipicidad generalizada se encuentra en los esquemas con los que organizamos nuestra experiencia vivida en una síntesis de reconocimiento. Esos esquemas son las tipificaciones que estructuran y organizan nuestra existencia mundana e incluso la experiencia pre-predicativa. El sentido común es, pues, un proceso constructivo,

10. Cfr. A. Schütz. «Fragments on the Phenomenology of Music». En F. Kersten (ed.) Music and Man, vol II, núm. 1-2 (1976) p. 11.

11. Cfr. A. Schüz. The Structures of the Life-World II. Bloomington: Northwestern Univ. Press, 1989 (3a ed.), p. 152-153. 
activo y no algo pasivamente dado, y la tipificación es un acto ideacional esencial. Gracias a ella, hacemos abstracción de lo específico y atendemos exclusivamente a la forma o al carácter estructural de los objetos.

Zaner opina que el tema fundamental de la obra de Schürz es el de la intersubjetividad ${ }^{12}$. En realidad, la sociología y todas las ciencias, en general, parten de la intersubjetividad como categoría fundamental de comprensión, pero no se la plantean como un problema. Cualquier interacción social deriva de la tesis general de la existencia del otro yo. Toda duda sobre el orro, sobre el mundo intersubjetivo, etc. deja intacta la creencia fundamental en el otro, en nuestro mundo y en sus objetos. Sin embargo, Schütz comprende que, para aptehender la intersubjetividad en sí misma, es preciso tematizar esa epojé de la epoje efectuada por el sentido común, abstenerse de participar en estas creencias y convertirlas en tema de investigación. Schütz no rehuyó nunca esta cuestión tan cara a la filosofía, porque no se contentaba con aceptar la experiencia aproblemática que tenemos de los otros en la vida cotidiana; le importaba, además, aclarar por qué podemos hablar de un otro en general y qué sentido le atribuimos a ese semejante que, a la vez, es un otro. Pensaba que la fenomenología transcendental no podía solucionar el problema de la intersubjetividad, ya que hacía del otro un elemento del mundo intencional constituido por el ego transcendental en el que todo alter ego recibe sentido y validez. ¿Como será posible la intersubjetividad si el otro y sus contenidos de conciencia son constituidos por el yo transcendental? Según Schütz, Husserl soluciona este problema en la esfera no reducida, pero no en la transcendental. Aún presuponiendo que los otros sean también yoes transcendentales, parece imposible solventar la contradicción entre los mundos transcendentalmente constituidos por ellos. Cada ego transcendental de la intersubjetividad debería ser constituido como hombre en el mundo y entonces se comprendería que todos contienen su propio ego transcendental. Esto sólo puede esclarecerse teniendo presentes las funciones de la intersubjetividad transcendental o de la comunidad monádica abierta. ¿Esta comunidad husserliana es, verdaderamente, una comunidad de hombres? ¿Cómo puede el yo declinarse transcendentalmente? ¿Es siquiera posible hablar de una pluralidad de egos transcendentales? Y si así fuera, ¿cómo la relacionaríamos con el eidos ego transcendental? ¿̨l alter ego sería tan sólo una posibilidad de dicho eidos?

En el Coloquio de Royaumont culminó públicamente el abandono schütziano de la fenomenología transcendental debido a que, según él, la reducción transcendental de Husserl no permite averiguar cómo mi yo transcendental sabe algo de un tú transcedental o cómo pueden fundar ese yo y ese tú transcendentales un nosotros transcendental ${ }^{13}$. Husserl habla de una estructura de yoes transcendentales o mónadas, que no son definidos. Por consiguiente, parece que el ego transcendental sólo puede concebirse en singular. Husserl no

12. Cfr. R.M. Zaner, "Theory of Intersubjectivity: Alfred Schütz". Social Research XXVII (1961), p. 71-93, p. 71.

13. Cfr. A. Schütz. "El problema de la intersubjetividad transcendental en Husseri», p. 311. 
aclara cómo es posible que la reducción transcendental (que constituye la intersubjetividad transcendental) sea practicada en comunidad. Además, las comunidades sociales no pueden ser consideradas como personalidades de un orden superior y no podemos encontrar en ellas niguno de los tasgos que revela el análisis de las personas individuales ${ }^{14}$. Husserl cometió la falacia de hipostasiar conceptos abstractos y atribuirles existencia personal.

Schütz no parece haber comprendido que, en la esfera reducida, el mundo entero es conservado aunque sólo en la medida en que es el correlato intencional de mi vida consciente. No meditó suficiente en la idea husserliana de que la subjetividad transcendental es en el fondo intersubjetividad transcendental que constituye el único mundo objetivo ${ }^{15}$; esa intersubjetividad transcendental se constituye en el ego meditante, pero de manera tal que es la misma para cualquier ser humano.

Schütz considera que en la $V$ de las Meditaciones cartesianas, Husserl no explica cómo es constituido el Otro como subjetividad transcendental, sino meramente como una unidad psicofísica mundana ${ }^{16}$; por consiguiente, Husserl no ha mostrado la posibilidad de un alter ego transcendental coexistente, constituido dentro del ego transcendental y esto sería necesario para superar el solipsismo de la esfera transcendental. Schütz piensa que la segunda eppojéno lleva a la constitución del Otro como mónada plena dentro de la mía, sino, en todo caso, a la apresentación de otro yo psicofísico a partir del mío ${ }^{17}$. Aun aceptando la constitución del alter ego transcendental por apresentación a partir de la experiencia de su cuerpo, aun presuponiendo la capa inferior preconstituida de las pertenencias propias, ¿para qué sirve la segunda reducción? ¿Como puede la apresentación del cuerpo orgánico extraño entrañar, a la vez, la apresentación de su mundo primordial? ¿Cómo la apresentación del otro yo psicofísico lleva a la concretización de una mónada completamente distinta? ¿Cómo se da entre mi mónada y la del otro constituido por mí una relación intersubjetiva y una comunidad transcendental? El ego transcendental constituye su mundo y a los otros según su ser y su sentido, los constituye sólo para sí y no para todos los egos transcendentales. Esto conduce a Schürz a afirmar que el otro ego del que habla Husserl se da como un ego psicológico y no como un ego constituyente de su propia esfera de propiedad. Por paradófico que parezca, Schütz intentó socializar al solitario ego transcendental, pero siguió convencido, como Hussert, de que sólo con relación al yo reciben los otros el significado específico que designamos con el término nosotros, sólo por referencia a ese nosotros cuyo centro soy yo, están otros en la situación vosotros y con referencia a ellos surgen terceros como ellos.

14. Cfr. Ibid, p. 313.

15. Un estudio más detallado del problema de ła intersubjetividad en Htusserl, Merleau-Ponty y Schütz y sus implicaciones en la fenomenología y la teorfa social, puede encontrarse en M. Carmen López Sáenz. Investigaciones fenomenologicas sobre el origen del mundo social. Zaragoza: PUZ, 1994.

16. Cfr. A. Schütz. Collected Papers I, p. 195.

17. Cfr, A. Schütz. Collected Papers III, p. 66-67. 
Nosotros creemos, como Carrington ${ }^{18}$ que Schitz no distinguió la esfera transcendental de la mundana en Husserl; para éste, la intersubjetividad debía ser explicada en ambas esferas. Sin embargo, Schütz sólo era sensible al problema de la intersubjetividad a nivel mundano y, por eso, interpretó la explicación de Husserl de la intersubjetividad transcendental como análoga a la intersubjetividad mundana y concluyó que ésta era absurda.

Schïtz considera que el problema epistemológico de la intersubjetividad está resuel to en la actitud natural; desde el punto de vista ontológico, la intersubjetividad es un presupuesto incuestionado en la esfera mundana. Su esencia es la simultaneidad (la conciencia de envejecer juntos), porque el fenómeno intersubjetivo no se da en mi duración interior, ni en el tiempo exterior de la naturaleza, sino en el tiempo objetivo o estándar medido por nuestros relojes y calendarios. No se trata de un tiempo construido arbitraria y subjetivamente, sino del tiempo común que coordina intersubjetivamente a los individuos. Schütz no nos explica por qué y cómo el tiempo estándar participa del tiempo cósmico, y no aclara en qué consiste éste. Tampoco explica esa mágica coincidencia entre el tiempo estándar, el tiempo cósmico y el tiempo interior. Por consiguiente, no está claro en qué radica la coordinación intersubjetiva de nuestros diferentes proyectos individuales.

No hay duda de que si hay un tiempo objetivo, éste estará íntimamente ligado a la intersubjetividad, pero, ¿será fruto de una mera convención? La respuesta podría ser aftrmativa por lo que se refiere al tiempo estándar, pero éste es reflejo del tiempo cósmico y, efectivamente, todos los sistemas temporales convencionales son de algún modo traducibles. No obstante, parece que la idea del tiempo cósmico sea un postulado que debemos presuponer, aunque no seamos capaces de captarlo tal y como es en sí mismo porque somos seres limitados temporalmente. Por otra parte, resulta difícil concebir el tiempo independientemente del espacio y, por ello, es casi imposible, comprender el tiempo objetivo, al margen de mi cuerpo y de su comportamiento.

Volviendo a Schuitz, y en consonancia con lo dicho, la situación más propiamente social es la situación cara a cara o relación-nosotros-pura, que se da entre los consoncios, entre quienes comparten una comunidad espacio temporal y son capaces de conectar sus motivos (los motivos-para del actor se convierten en motivos-porque del partícipe), ya que comparten la vida interior en curso del otro. Sólo en las relaciones cara a cara el otro es aprehendido como individuo único en su situación biográficamente determinada. En cualquier otra dimensión del mundo social, el otro es experimentado y aprehendido como un tipo. El resto de situaciones sociales son variaciones de ella y se deducen de la combinación de las dos principales características de la relación-nosotros: la inmediatez y la reciprocidad. En términos husserlianos,

18. Cfr. J. Carrington. «Schutz on transcendental Intersubjectiviry in Husserln. Human Studies. vol. 2 (1979), p. $95-110$. 
en la situación cara a cara se produce una intencionalidad directa ${ }^{19} \mathrm{y}$, por consiguiente, en ella se logra la mayor evidencia.

En Schütz, la orientación hacia el tú es el prerrequisito de toda acción social, aunque para convertirse en relación social es preciso que el yo actúe sobre el otro, que se produzca una relación-nosotros. El acceso al otro se basa en la interpretación de las experiencias almacenadas en nuestro stock de conocimientos y en la presuposición de que también son aplicables a otro. Percibimos al alter ego sin necesidad de reflexión; por apresentación, como un ser dotado de una conciencia similar a la mía que da significado subjetivo a sus acciones ${ }^{20}$. Pero el acceso a la vida interior del otro no puede ser total, porque si lo fuera seríamos la misma persona. La comprensión del otro es, pues, en la sociología de Schütz, un límite nunca alcanzado, pero que da forma a la intención denominada orientación bacia otro. Para logtar esta comprensión, Schütz construye unas categorías y modelos basados en la experiencia de sentido común, precientífica, de la realidad social. Pero no se cuestiona cómo es posible que nutestras experiencias, que se suponen referidas a ottos concretos, conduzcan a la convicción de su existencia. Schürz decide comenzar por la experiencia de la conciencia intencional del otro, es decir, por la orientación hacia el otro en la que se parte de su existencia como una persona humana concreta y no como un otro transcendental. Siguiendo la tradición fenomenológica, recurre a la analogía y a la empatía para establecer la idealización del puedo volver a hacerlo y la del y así sucesivamente y para comprender los significados que el otro da a sus acciones, pero reconoce que su mundo transciende el mío porque nuestros sistemas de relevancias, vivencias, biografias son distintos y sólo podemos conocer el interior del otro desde fuera. Esto indica la finitud de mi conocimiento, pero también demuestra la otredad y la diferencia de las conciencias. Sin embargo, Schütz piensa que el yo es autotransparente, cuando la experiencia nos dice que, en ocasiones, el otro ayuda al yo a comprender mejor el significado de sus acciones, la imagen de la parte invisible de su propio cuerpo, sus motivos inconscientes. Para que el yo sea verdaderamente autotransparente es necesario que previamente se reconozca como yo transcendental. Del mismo modo, para comprender verdaderamente al otro no debo limitarme a la autointerpretación. ¿Bastará la transferencia empática? Schütz considera que ésta descubre únicamente la conciencia propia y no la conciencia del otro. Critica la indefinición hussetliana del término 21 y nos insta a no partir de la semejanza con el yo, sino de la tesis general del tú como un yo extraño, para afirmar luego que todo tú constituye sus vivencias de conciencia del mismo modo que lo hace la mía, que su corriente de pensamiento muestra la misma

19. Husserl distinguia una intencional longitudinal (las vivencias en su desarrollo o vivencias prefenoménicas, es decir, no zematizadas) y una intencionalidad transversal (la reflexión posterior sobre dichas vivencias). Cf. E. Husserl. Lecons pour une phénoménologie de la conscience intime du temps. París: PUF, p. 155-156.

20. Cfr. A. Schütz. Life Forms and Meaning Structure. Londres: Routledge and Paul Kegan, I982, p. 130-131.

21. Cfr. A. Schütz. Collected Papers $M I$, p. 37. 
estructura que la mía; es decir, que el otro es como yo, capaz de actuar y de pensar. En este sentido, la teoría de la intersubjetividad de Schütz cae en una especie de psicologismo mitigado.

Cuando Schuitz asegura que lo que determina la intersubjetividad es la experiencia vivida del tú y la Du-Einstellung, parece afirmar que la interacción social está basada en una experiencia preconceptual y que siempre cuenta con una dimensión no conceptualizable. Nosotros, siguiendo a Husserl, creemos que esta experiencia, al igual que la tesis general que afirma la existencia del mundo y de los otros es fruto del purto de vista natural, pero éste, a su vez, está ya marcado por la reflexión y por la constitución del sentido, aunque en el ámbito mundano se haga epojé de esos condicionamientos.

A pesar de las críticas dirigidas a la intersubjetividad husserliana, Schütz estaba de acuerdo con algunas de sus conclusiones, por ejemplo, su doctrina sobre el cuerpo y la percepción, sobre el espacio y el tiempo de los sujetos, la intercambiabilidad de las perspectivas, la tipificación e idealización, la apresentación de la conciencia ajena, el establecimiento de un entorno común comunicativo, la prioridad de la comprensión intersubjetiva y la intencionalidad sobre la comunicación, etc. Schütz entendió la intersubjetividad como categoría ontológica fundamental de la existencia humana y como fundamento de la sociabilidad; buscó en la fenomenología una solución para ella, pero no consiguió resolver los problemas de la teoría husserliana de la intersubjetividad transcendental, sino que se limitó a disolverlos y a analizar la intersubjetividad empírica. Olvidó que Husserl acunó el término intersubjetividad transcendental para referirse, no a las interrelaciones humanas fácticas, sino a la esencia de toda comunidad humana real o posible. ¿Qué significa esta intersubjetividad transcendental por oposición al concepto mundano de intersubjetividad? Podríamos decir que, para Husserl, es la estructura básica que define a la humanidad y que origina la intersubjetividad fáctica y las categorías que sirven para aprehenderla. Por consiguiente, creemos que este concepto tiene sentido y puede incluso resultar operativo para analizar la noción de intersubjetividad por la que se interesa Schütz, esa que funda todas las otras relaciones humanas y sociales.

Como el mismo Schütz reconoce: "Dentro de la subjetividad transcendental del filósofo que medita, subjetividad que sólo debe valer para él, la creación del universo de las mónadas y del mundo objetivo para todos se muestra imposible bajo cualquier hipótesis. Pero el esclarecimiento de la estructura de sentido de la intersubjetividad y de la validez del mundo para mí en tanto objetivo, es y sigue siendo una tarea legítima del análisis fenomenológico de la constitución. Y Husserl no sólo indicó esta tarea como terreno de investigación, sino que en gran parte la realizó" ${ }^{22}$.

Siguiendo al maestro, Schütz asegura que la intersubjetividad y la objetividad del mundo son igualmente originarias y, por lo tanto, la una no puede ser derivada o ser construida por la otra. 


\section{Constructivismo, realismo o idealismo fenomenológico}

Se ha acusado a la fenomenología de reificar el noema, de pensar que éste es algo real en el mundo y no el mero correlato de una noesis y que, por encima del mundo intencional (mundo de noemata), hay un mundo de cosas en sí cuya existencia es indudable ${ }^{23}$. Esta idea, más metafísica que fenomenológi$\mathrm{ca}$, hace que no podamos considerar a Schütz y a los fenomenólogos, propiamente hablando, como constructivistas, es decir, Schütz no es un teórico que concibe la sociedad como como un compuesto de significados, acciones e intenciones humanas, organizados de forma que la realidad social resulta ser un producto de definiciones individuales y colectivas. Sólo si descontextualizamos algunas de sus afirmaciones ${ }^{24}$, lo catalogaremos de esta manera.

La fenomenología asegura que lo que constituye la realidad es el sentido de nuestras experiencias y no la estructura ontológica de los objetos, pero esto no significa que el sistema de Schürz sea idealista, porque lo verdaderamente real, para él, es el sentido; sin él no tendráamos conciencia ni conocimiento de las acciones ni de los objetos. Lo que distingue a las ciencias sociales de las naturales es que la materia de aquéllas tiene ya un sentido constituido en su objeto mismo (la vida social); elegimos entre diferentes interpretaciones de los objetos, pero los sentidos de éstos están ya presentes en el mundo social, es decir, el sociólogo no se inventa nada, sino que se limita a comprender en profundidad la vida humana-social; para ello aplica categorías y conceptos específicamente construidos para aprehender con rigor esa realidad. El propio Schütz sitúa la fenomenología entre el idealismo y el realismo ${ }^{25}$ y la considera principalmente como un método científico.

Pensamos como Spurling que la fenomenología de Schütz contiene rasgos positivistas ${ }^{26}$; esto implica que su programa no es abiertamente constructivista; es decir, que Schütz no entiende la ciencia social fenomenológica tan sólo como una construcción de tipos ideales y modelos cientificos del mundo social: "construccionismo de la ciencia social en el interior de una pura estructura de modelosn ${ }^{27}$.

Schütz practica la epojé y su construccionismo sería, en todo caso, metodológico y no ontológico, porque él no es un ontologista dogmático. Su aproximación a la realidad social es puramente metodológica y esto posibilita la neurralidad de su concepción de la ciencia. Transformamos y reorganizamos el mundo social al mismo tiempo que lo interpretamos; fabricamos nuestro mundo construyendo su sentido y eso no implica que lo tergiversemos.

23. Véase, por ejemplo, A. Schütz. Life Forms and Meaning Structure, p. 103-105.

24. Por ejemplo, Schütz afirma: "Hablamos de ámbitos de significado y no de sub-universos, porque es el significado de nuestra experiencia y no la estructura ontológica de los objetos lo que constituye la realidad". (A. Schütz. Collected Papers I, p. 230.)

25. Cfr. A. Schürz. El problema de la realidad social Buenos Aires: Amorrortu, 1962, p. 105.

26. Cfr. L. Spurling. Phenomenology and social World. Boston; Routledge and Paul Kegan, 1977, p. 174.

27. Cfr. B.C. Thomason. Making Sense of Reification. Londres: MacMillan Press, 1982, p. 62 . 
Ciertamente, el método de Schütz está cerca del constructivismo, pero no tiene como misión clarificar la estructura ontológica del ser, sino estudiar la percepción de la realidad fenoménica y su influencia en las acciones humanas. Esto no significa que Schütz identifique por completo la esfera ontológica con el significado que le otorgamos; el hecho de que centre su análisis en nuestra experiencia de la realidad no quiere decir que no haya nada más allá de ella. No obstante, Schütz tendría que haber profundizado más en el concepto fenomenológico de experiencia y haberla deffnido con rigor para soslayar ese tipo de acusaciones.

Hemos visto que Schütz radicaliza la comprensión de Parsons y hace de ella una verdadera comprensión subjetiva. Reconoce que hay diversos grados en ella y que la comprensión nunca puede ser total, ya que esto implicaría que el yo y el otro tienen una corriente de conciencia común. Esto no es un obstáculo para la ciencia social, puesto que para comprender la acción del otro me basta conocer sus motivos típicos. Schitzz demuestra que es posible una ciencia de lo subjetivo y que ésta no se limita a reconstruir arbitrariamente las acciones sociales, sino que actúa comprendiendo. De ahí que et Verstehen no se entienda tanto como un método, cuanto como el modo en el que el hombre experiencia el sentido mundo cotidiano. Que la comprensión sea subjetiva no significa, pues, que los motivos de las acciones sean privados e incontrolables, sino que la meta schütziana es clarificar lo que el actor significa con su acción sin imponerle los esquemas interpretativos del observador (aunque, evidentemente, los estudios de éste también aumentan el grado de conocimiento que el propio actor posee). Asf pues, el Verstehen schütziano es prioritariamente un modo del sentido común de experienciar los asuntos humanos; después es un problema epistemológico, y, finalmente, un método peculiar de las ciencias sociales. Los ricos análisis del segundo aspecto efectuados por la fenomenología husserliana arrojaron luz sobre el primero y el tercero. Schütz se nutrió de ellos, especialmente de la teoría fenomenológica de la percepción. De ahí su rechazo de la teoría causal de la percepción: ésta dice que el objeto físico y la experiencia que tenemos de él están conectados de tal forma que, si pudiéramos obtener una relación extensional de la percepción, seríamos capaces de explicar (desde el punto de vista de una tercera persona) la identidad del objeto percibido por distintos sujetos. Esta teoria no explicita suficientemente la razón de la objetividad de las cosas, es decir, la recognición por parte de un sujeto de que su objeto es un Objeto experimentado por otros. La objetividad de los Objetos, su ser Objetos compartidos por una pluralidad de diferentes sujetos, puede ser comprendida sólo si reconocemos el papel que juega el encuentro cultural y comunicativo entre sujetos. Efectivamente, el reconocimiento de que el objeto que yo experimento es el mismo que experimenta el otro y de que, por tanto, es un verdadero Objeto, sólo puede ser establecido gracias al hecho de que el yo y el otro participan de los mismos constructos culturales y se comunican entre sí; merced al significado de este encuentro en el que se intercambian descripciones de los respectivos objetos, se busca el consenso entre los sujetos sobre qué sea un Objeto. Por consiguiente, la constitución 
del mundo Objetivo es un logro intersubjetivo, asentado en la conexión de los sujetos que participan en el encuentro comunicativo.

Aunque, en efecto, el mundo intersubjetivo es un conjunto de construcciones y tipificaciones y lo dado es, al mismo tiempo, construido por la conciencia como típico, a pesar de que los actos originados desde las tipificaciones tienen la misma fuerza que los conceptos a priori o los universales, ni Schürz, ni la fenomenología reducen el mundo a una pura construcción de significados subjetivos o carecen - como se ha dicho- ${ }^{28}$ de un concepto sólido de realidad objetiva; lo que rechaza la fenomenología es el objetivismo de la ciencia moderna y su pretensión de saber absoluto, pero aspira a la objetividad y, para ello, aporta una concepción de la verdad como evidencia impletiva o intencionalidad plena, que es incompatible, tanto con el realismo como con el constructivismo.

Buena parte de los críticos de Schütz no tienen en cuenta que el concepto de realidad que éste maneja es intencional y que la fenomenología no construye ex nihilo el mundo, sino que el sujeto transcendental se co-constituye con él y está constantemente referido a él. Decir, con Schürz, que la realidad es la experiencia de la realidad es afirmar que todas las percepciones que podemos tener están referidas a una unidad y que esta implicación se debe a unos esquemas determinados. Cuando Husserl y Schütz hablan de constitución se refieren a estos esquemas de implicación o familiaridad que dirigen la expe. riencia. Este es el significado del a priori de correlación entre la subjetividad de la conciencia y el mundo. La reducción pretende recuperar esa vida constituyente transcendental, generalmente anónima, que da al mundo el único sentido que éste puede tener para nosotros. Este a priori de correlación no significa sólo que los objetos se constituyen o son correlatos de la vida subjetiva o intersubjetiva, sino que cada objeto tiene un modo peculiar de experiencia en la que se dan los rasgos típicos de cada ámbito del mundo. Se podría objetar que el análisis del modo de darse la realidad humana no alcanza su esencia; Husseri respondería que, para investigar las propiedades de las objetividades de cada ontología regional empleamos la variación imaginativa y que dichas ontologías deben guiar a las ciencias, no sustituirlas. Schütz acepta la tesis de Fink de que Husserl evitó los problemas ontológicos y se ocupó únicamente de las ontologías formales y regionales.

En todo caso, el constructivismo de Schütz sería únicamente metodológico. Para ser más precisos, Schütz dialectiza el realismo y el constructivismo; afirma que nos relacionamos con el mundo pasivamente (descubriéndolo) y activamente (constituyéndolo) y esto da lugar a dos ontologías distintas, pero complementarias. De este modo, Schütz nos invita a desarrollar una dialéctica positiva de las relaciones humanas, a combinar el sentido activo y el pasivo de la realidad social, porque el significado que damos a la realidad transforma y constituye un mundo al mismo tiempo que lo descubre e interpreta. De ahí

28. Cfr. G. Gummer. "A critical Examination of Phenomenological Sociology". Sociological Analysis IYI, I (1972), p. I3. 
la distinción schütziana entre nuestro mundo y nuestro mundo. Por todo lo dicho, la sociofenomenología de Schütz sólo es constructivista en un sentido relarivo (el mismo que puede servir para considerarla positivista y realista), por cuanto su objeto es la realidad social y son los sujetos los que le dan sentido, pero no sin que la propia realidad se lo proponga.

\section{Conclusión}

Como tantos otros seguidores de Husserl, Schütz mostró que la fenomenología no es un método idealizante y solipsista, sino que, por el contrario, resulta imprescindible para estudiar comprensivamente la realidad social y para tematizar su punto de partida: las relaciones entre los sujetos. Su gran mérito fue haber descubierto en su profundidad, las presuposiciones, estructura y significación del mundo del sentido común, de ese mundo intersubjetivo experimentado por el hombre en la actitud natural. Su pormenorizada descripción de éste hace patente la aplicabilidad empírica y la riqueza de los conceptos fenomenológicos.

No obstante, al reducir la fenomenología a psicología transcendental, Schütz se encuentra con dificultades para justificar la constitución de un mundo objetivo común. Se apoya en la creencia ingenua de la existencia efectiva del otro y cae en el psicologismo, porque confunde el mundo original de la experiencia con el mundo de la vida cotidiana o mundo de las interpretaciones del sentido común ${ }^{29}$ y porque reduce el ego transcendental al psicológico; combina dos constituciones, la transcendental y la psíquica, y dos egos constituyentes, y transforma la diferencia entre lo transcendental y su correlato transcendente, entre filosofía y ciencia positiva, en una simple distancia entre lo profundo y lo superficial. Este psicologismo dificulta la derivación que hace Schütz de la estructura básica del mundo social (el mundo que existe para mí en tanto ego transcendental) a partir de nuestro propio pensamiento cotidiano sobre los prójimos. De ahí que algunos autores hayan llegado incluso a decir que: "La ciencia social de Schütz no está fundada o basada en una fenomenología y que su metodología no es más que un individualismo idealistan ${ }^{30}$, cuyo único objeto es la reducción del mundo objetivo al comportamiento de los individuos. El privilegio que Schütz concede a las relaciones cara a cara hace pensar en una cierta reducción de la realidad social a un tejido de relaciones interindividuales, pero nosotros opinamos que no puede atribuirsele tal reducción, a pesar de que - como él- pensamos que sólo dichas relaciones aclaran el origen y el verdadero sentido de la sociabilidad. Por este motivo, podríamos decir que el análisis de Schütz es enriquecedor, pero requiere otros estudios complementarios. Además, al privilegiar la experiencia social directa $y$, por

29. Cfr. H. Hindess. Phenomenology and Methodology in the social Science. University Harvester Press, 1977, p. 63.

30. Cfr. H. Hindess. "The phenomenological Sociology of Alfred Schutz. Economy and Society 12, núm. l (1972), p. 15. 
consiguiente, las relaciones cara a cara, Schütz da muestras de un positivismo $y$ un realismo alejados del constructivismo que se le imputa. No hay que olvidar, sin embargo, que la meta de Schütz no es el estudio de los objetos mismos, sino de su significado constituido por las acrividades de nuestra conciencia. En su opinión, el sociólogo observa los hechos de la realidad social, acota su objeto de estudio, crea modelos, etc. Es cierto que también selecciona una serie de significados para sus actores, pero sólo cuando estos significados reproducen el comportamiento observado en determinada situación. Así se obtienen dos resultados fundamentales: relacionar la situación con el significado que le otorga el actor que la ejecuta y explicarla. Por este motivo, no podemos decir que la interpretación de la realidad social de Schütz sea reduccionista, sino todo lo contrario: la sociofenomenología considera la sociedad como un todo formado por seres humanos capaces de crearla gracias a sus producciones y a sut donación de sentido.

Por otra parte, se ha vinculado la sociofenomenología a las teorías accionistas y esto se debe a lo que, para nosotros, es un defecto del pensamiento de Schütz: la prioridad otorgada a los procesos de significado activos. Conocemos la importancia que Husserl concedía a la percepción y sabemos también que la relacionaba estrechamente con la reflexión. En cambio, Schütz la entiende como una recepción de datos pasiva. Por eso en "Type and eidos" habla de "percepción pasiva, sufrida", etc. Pero si los datos se reciben pasivamente y el sentido se origina únicamente en los procesos mentales activos, ¿como puede hacerse presente a la conciencia el sentido noemático en el caso de una percepción no activa? (digamos de paso que dicho sentido es necesario para reconocer las tipificaciones de la experiencia y su intencionalidad). Schütz respondería que la única fuente de dicho sentido es el stock de conocimientos a mano. Por consiguiente, los procesos de constitución del sentido, la intencionalidad, etc. no serían esenciales. Bastarían los procesos pasivos y el stock de conocimiento se convertiría en el repositor de tipos sedimentados, necesario para dar significado a las experiencias posteriores. Sin embargo, no está claro que los tipos ideales puedan desprenderse de la actividad reflexiva y convertirse en topicos, automatizarse. Quizás esto sea habitual en el uso que el hombre del sentido común hace de ellos, pero no lo es para el científico social. Además, todo tipo ideal es el correlato noemático de un proceso de conocimiento noético. Schütz piensa que este último puede darse pasivamente; nosotros creemos que es imposible y que, en todo caso, habría sido conveniente que Schütz aclarara mejor las relaciones entre el científico social como observador y como teorizador. Husserl distinguía la formación de juicios genéricos de universales empíricos contingentes de la intuición del eidos por ideación y libre variación. Schütz piensa que esta ideación no puede revelar nada que no esté ya preconstituido en el tipo que recibimos de los objetos familiares ${ }^{31}$. Es decir, Schürz afirma que los conceptos eidéticos no se constituyen en la conciencia, sino que forman parte de una ontología ya dada y simplemente se nos imponen. Las tipificaciones se

31. Cfr. A. Schütz. "Type and eidos...", p. 147. 
fundan en la experiencia pre-predicativa y los conceptos eidéticos se basan en ellas. Los tipos son procesos pasivos dados. En suma, Schütz presupone el carácter ontológicamente prioritario del mundo real.

Algunas de estas limitaciones pueden deberse a que el estudio de Schütz no va más allá de los materiales publicados de Husserl. En sus últimos escritos, Husserl intento superar algunas de estas aporías; aludió a una vida original que no era ni una ni múltiple, ni factual ni esencial, sino el fundamento último de estas diferencias y se refirió a la Lebenswelt física y social como a priori último; por otro lado, Husserl continuó ocupándose ampliamente de la intersubjetividad transcendental, la ética, la historia, etc.

Habermas afirma que Schütz y Husserl se quedaron en una simple generalización de la propia experiencia ${ }^{32}$ porque no alcanzaron a ver que el único medio en el que se cumple la dialéctica de lo particular y lo universal es el lenguaje ${ }^{33}$. Nosotros, en cambio, estamos convencidos, como aquéllos, de que el encuentro comunicativo que origina el consenso se funda en el reconocimiento previo del otro como sujeto, como individuo humano que está intencionalmente relacionado con los objetos y cuyo comportamiento indica las cosas que experimenta. Es decir, Verstehen no es tan sólo, como quiere Habermas, comprensión comunicativa.

El problema es que Schütz no presta demasiada atención a la génesis fenomenológica del mundo social y prescinde de la fenomenologfa transcendental; aunque dilucida las estructuras antropologicas de la comprensión y el estatuto epistemológico de las ciencias sociales, se ve abocado al relativismo y al constructivismo, porque renuncia a buscar criterios para constrastar las interpretaciones y evaluar su acercamiento a la verdadera realidad social. Schütz hace un análisis descriptivo, tipológico del mundo social, pero no distingue adecuadamente la actitud que adoptamos en la vida cotidiana, de las estructuras constitutivas del mundo social que no se nos dan en esa vida. Quizás por eso no resuelve completamente el problema de la comprensión del otro ni el del objeto cultural como hueila de su vida consciente.

No basta con explicitar las estructuras fundamentales del mundo vivido, ya que éstas no existen per se. Schütz se aparta de la teoría de la constitución transcendental husserliana por considerar que ésta se transformó, en Husserl, pretendiendo llegar a ser, más que la efectuación de una exégesis del ser, el fundamento de su estructura ${ }^{34}$. Nosotros creemos que Husserl se mantuvo fiel a sus principios y que nunca pretendió deducir la intersubjetividad de la subjetividad transcendental, sino explicitar el sentido de la existencia verdadera de los otros; del mismo modo que la conciencia de sí y la conciencia del otro son inseparables, no puede haber prioridad ontológica de la constitución del yo en relación con la del otro ni viceversa. En realidad, las aporías a

32. Cfr. J. Habermas. La logica de las ciencias sociales. Madrid: Tecnos, 1988, p. 198.

33. Cfr. Ibid, p. 198.

34. Cfr. A. Schutz. "El problema de la intersubjetividad transcendental en Husseri", p. 315-316. 
las que conduce la intersubjetividad transcendental husserliana expresan la esencia misma de nuestra situación; por eso creemos que no hay que abandonarla y que tiene un gran interés para las ciencias humanas, porque analiza la genesis del sentido y del reconocimiento del significado. Si dejamos de lado estos problemas, resulta inexplicable la comunicación. Tal vez tengan razón los críticos cuando dicen que Schütz no fue plenamente consciente de sus presupuestos filosóficos o no supo sacar de ellos todas sus consecuencias, pero, al menos, contó con la filosofía y creó una escuela de investigación interdisciplinaria muy significativa por lo que a las ciencias humanas se refiere. Sus análisis han hecho posible una rica definición de la tarea de comprensión, una sistematización del método de los tipos ideales y un esclarecimiento del postulado weberiano de la adecuación significativa. Schütz nos ha demostrado que la fenomenología es un instrumento adecuado para la conceptualización y que suministra un contenido más rico a los tipos ideales en la medida en que hace aparecer las estructuras fundamentales de la existencia social a partir de las cuales se construyen aquéllos. Estos resultados deberían penetrar también en el resto de las ciencias humanas.

Por su parte, la interpretación de Husserl nos sigue invitando a comprender al otro y al yo más allá de sus determinaciones psicológicas y sociales, a buscar una esencia de ambos que sirva de sustrato a sus realizaciones empíricas, pero que no se reduzca a ellas. El padre de la fenomenología nos enseñó que la sociabilidad (esa concreción vital de la intersubjetividad) no puede entenderse si antes no se ha comprendido la intersubjetividad. Si admitimos que ésta se gesta transcendentalmente y forma parte de la propia subjetividad, la sociedad puede dejar de entenderse como una creación arbitraria que sirve para remediar la rapacidad y el egó́smo del sujeto. Husserl demostró que el utilitarismo estratégico no es la única forma de racionalidad en la que podemos pensar.

Por todo lo dicho hasta el momento, creemos que la fenomenología presta unidad a las ciencias fragmentadas, ya que es capaz de fundamentarlas sobre unos principios antropológicos básicos y una metodología ontológica determinada. Para ello, será preciso asumir la racionalidad estructural de todos los objetos del conocimiento humano, el concepto de mundo de la vida o correlato de la conciencia existente y la intersubjetividad radical de dicho mundo. La fenomenología puede ofrecer a las ciencias significados y vías de intercomunicación práctica, pero para ello debe dialogar con ellas e intentar ponerlas en contacto en torno a una preocupación fundamental y común: las raíces del hombre y de la condición humana. En este sentido, A.T. Tymieniecka ha propuesto una fenomenología del hombre y de la condición humana como factor fundacional para una comunicación interdisciplinaria ${ }^{35}$. La fenomenologia no nos propone una sociología determinada, sino una reinterpretación crítica y constructiva de las investigaciones sociológicas. Para el fenomenólogo, lo

35. Véase, por ejemplo, A.T. Tymieniecka. The Phenomenology of Man and of the human Condition. Analecta Husserliana XIV. Dordrecht: Kluwer Ac. 1983, p. 40. 
social nunca puede ser un objeto; se aprehende viviéndolo, describiendo adecuadamente lo vivido para reconstruir su sentido. La sociofenomenología no dicotomiza al sujeto de su sociedad, ya que es aquél el que otorga significado a aquélla. Por otro lado, la noción fenomenológica de intencionalidad ha sido de suma utilidad para la sociología. La fenomenología enfatiza la libertad, el protagonismo en la vida social del sujeto y de su acción (a diferencia del excesivo determinismo que se desprende del funcionalismo social). Considera que el individuo no es simple resultado de la acción social, sino que constituye el principio de ésta; se interesa por la realidad construida, por la génesis de las normas, por la creación, por la microsociologia, por la interacción individual $\mathrm{y}$, ante todo, por el sentido. 